\title{
Measurement and Modeling of Subway Near Shadowing Phenomenon
}

\author{
Ke Guan*, Zhangdui Zhong*, Bo $\mathrm{Ai}^{*}$ and Cesar Briso-Rodríguez ${ }^{\dagger}$ \\ ${ }^{*}$ State Key Laboratory of Rail Traffic Control and Safety \\ Beijing Jiaotong University, 100044, Beijing, China \\ ${ }^{\dagger}$ Escuela Universitaria de Ingeniería Técnica de Telecomunicación \\ Universidad Politécnica de Madrid, 28031, Madrid, Spain \\ E-mail: myecone@hotmail.com
}

\begin{abstract}
This paper focuses on one vital aspect in propagation characteristics inside subway tunnels: near shadowing phenomenon in a practical environment. In order to characterize this effect, an accurate measurement has been made at 2.4 GHz in a real environment in Madrid subway. By analyzing the numerical results in this measurement, the characteristic of near shadowing phenomenon in propagation process has been revealed and corresponding engineering suggestions have been given in order to compensate the near shadowing effect. Finally, statistical model including the depth, duration and length of near shadowing, fast fading and attenuation inside wide tunnel and narrow tunnel has been built and simulated.
\end{abstract}

Index Terms-measurement; modeling; near shadowing; propagation; tunnel

\section{INTRODUCTION}

The rapid growth of railway all over the world requires high quality radio communications to provide necessary capacity and reliability of railway [1]. Among all the cases of the railway environments, tunnel is a typical scenario, which exists in metropolitan cities and mountainous areas. Since seventies of last century several reports of measurements and modeling regarding the propagation characteristics inside tunnels have been published [2] - [6]. Nevertheless, except those measurements reported in [2], very few measurements were carried out in a realistic environment with real trains. In fact there are considerable differences between measurements carried out in realistic (real tunnel, with train) and non-realistic environment (simulative tunnel, without train), since some important phenomena of propagation in subway operation can be observed only in a real environment with real train. The most typical one of the important phenomena is that when the train is passing the transmitter the received signal power of referenced configured antennas (one head and one tail) on train suffers a strong fading.This phenomenon affects propagation severely in a realistic tunnel with real antennas configurations on train; however it has never been focused on by any prior dissertation.Only [7] mentioned this phenomenon but never systematically analyzed or modeled it.

This work is supported by the Joint State Key Program of the National Natural Science Foundation of China (No. 60830001), the State Key Laboratory of Rail Traffic Control and Safety (No. RCS2008ZZ006), Beijing and the Program for Changjiang Scholars and Innovative Research Team in University under Grant No. IRT0949. Jiaotong University.

CHINACOM 2010, August 25-27, Beijing, China

Copyright $\odot 2011$ ICST 973-963-9799-97-4

DOI 10.4108/iwoncmm.2010.6
To fill this researching gap and to gain deeper insight and better understanding of this phenomenon, we have made a measurement in $2 \mathrm{~km}$ tunnels with 4 base transmitters and 3 train stations using standard subway trains in Madrid, in which this phenomenon has been revealed. Since it occurs when the train is near the transmitter, we name it as Near Shadowing Phenomenon.

Then based on the measurement, numerical analysis of near shadowing phenomenon characteristics have been made; corresponding engineering suggestions have been given in order to compensate the attenuation and improve the performance of the current referenced antennas configurations on train. Finally, statistical modeling and comparison towards near shadowing effect in double track tunnel (9.8 m width) and single track tunnel ( $4.8 \mathrm{~m}$ width) have been made.

\section{Measurement in Operational Madrid Subway}

\section{A. Measurement environment}

Measurements have been carried out in a typical line with a high traffic density on the subway tunnels of Madrid in Spain. Fig. 1 shows the dimension and the skeleton map of the tunnels: Tribunal-Principe Pio, where a $1695 \mathrm{~m}$-long track has been selected for measuring with one station on the middle and one single track tunnel. The test has used four $2.45 \mathrm{GHz}$ transmitters installed at 455, 664, 946 and $1228 \mathrm{~m}$. The tunnel used in measurements and the transmitter fixed inside tunnels can be seen in Fig. 2, respectively.

\section{B. Test system}

The system was composed of four base transmitters and one mobile receiver installed on the train. The test transmitters are proprietary portable equipment that covers the $2.4 / 2.5-\mathrm{GHz}$ band in $1 \mathrm{MHz}$ steps with an output power of $20 \mathrm{dBm}$ and has a 3-dB power splitter to feed two antennas.

The antennas used in all transmitters were $8 \mathrm{dBi}$ gain flat antenna with $65^{\circ} \mathrm{E}$ and $\mathrm{H}$ main lobe and circular polarization. Inside the tunnel, the antennas were located $4 \mathrm{~m}$ over the floor and $25 \mathrm{~cm}$ from the tunnel walls.

The measurement equipments were deployed in the locomotive cabin of the train. The trains used were standard Madrid subway trains with four cars and $60 \mathrm{~m}$ length. The receiving antennas were vertical polarized antenna with $8 \mathrm{dBi}$ Gain and 


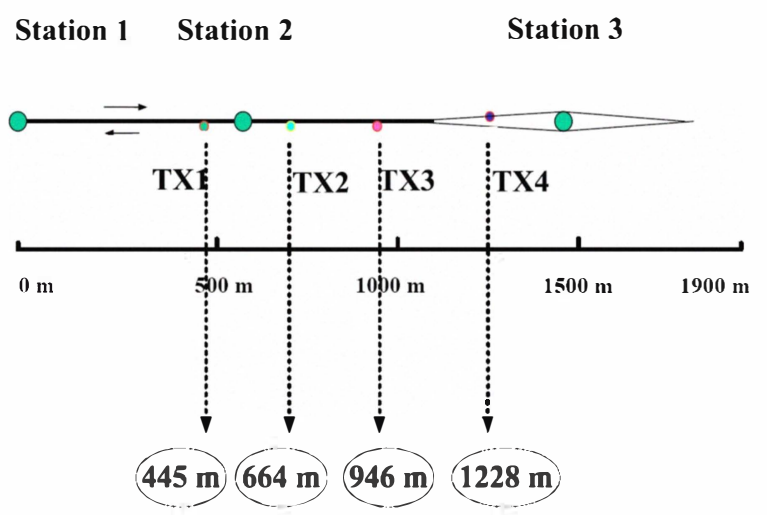

Fig. 1. Skeleton map of tunnel: Tribunal-Principe Pio.

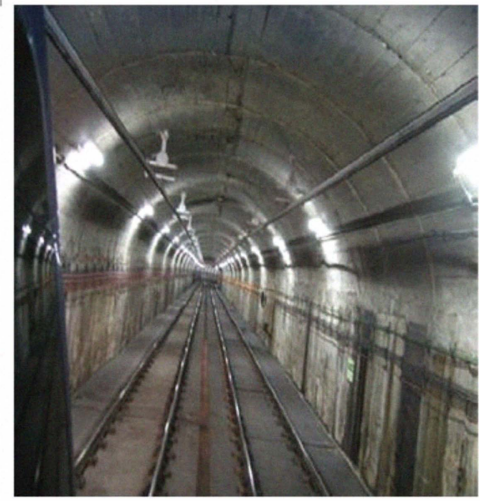

(a)

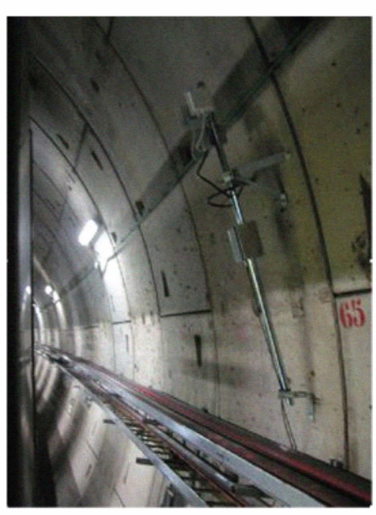

(b)
Fig. 2. (a) Photo of classical arched tunnel used in measurements. (b) Photo of transmitter fixed inside tunnels.

$65^{\circ} \mathrm{E}$ horizontal and vertical beam width. Near each antenna there was a $30 \mathrm{~dB}$ preamplifier to improve noise figure and to reduce coaxial feeder loss. Finally, we got a rate of one measurement per $1 / 2$ wavelength. All the detailed parameters of the measurements have been supported in Table. I.

\section{Numerical Analysis And Modeling OF NeAR SHADOWING PHENOMENON}

\section{A. Measurement results and analysis}

In the measurement, configuration of antennas on train uses two antennas, one installed in the front and one in the rear part of the train. This configuration is easy to implement and provide good results. Therefore, it is the referenced antennas configuration used in current real subway systems.

The measurement results can be seen in Fig. 3, Fig. 4 and Fig. 5. We can see the power average along the test tunnels with four transmitters, each single one has a different frequency and different location along the track. With these results we can conduct a statistical model and analysis on near shadowing phenomenon in wide (dual track) and narrow (single track) tunnels.

In this measurement, a very important phenomenon has been observed when the train is in front of the transmitter the
TABLE I

MEASUREMENT ENVIRONMENT AND TEST SYSTEM

\begin{tabular}{|c|c|c|}
\hline $\begin{array}{l}\text { Tunnel interval } \\
\text { Tunnel type }\end{array}$ & \multicolumn{2}{|c|}{$\begin{array}{c}\text { Tribunal-Principe Pio } \\
\text { Arched }\end{array}$} \\
\hline Tunnel dimension & \multicolumn{2}{|c|}{$\begin{array}{c}\text { Width: } 9.6 \mathrm{~m} \text {; Wall height: } 3.75 \mathrm{~m} \text {; } \\
\text { Roof height: } 6.1 \mathrm{~m}\end{array}$} \\
\hline Train length & \multicolumn{2}{|c|}{$60 \mathrm{~m}$} \\
\hline Train speed & \multicolumn{2}{|c|}{$20 \mathrm{~km} / \mathrm{h}$} \\
\hline Testing frequency & \multicolumn{2}{|c|}{$2.4 \mathrm{GHz}$ (CBTC) [1] } \\
\hline Sampling interval & \multicolumn{2}{|c|}{$0.5 \lambda$} \\
\hline $\begin{array}{l}\text { configuration } \\
\text { of antennas }\end{array}$ & $\begin{array}{l}\text { Transmitting } \\
\text { antenna }\end{array}$ & $\begin{array}{l}\text { Receiving } \\
\text { antenna }\end{array}$ \\
\hline Location & $\begin{array}{l}4 \mathrm{~m} \text { over ground } \\
25 \mathrm{~cm} \text { from wall }\end{array}$ & on the cabin \\
\hline Gain & $8 \mathrm{dBi}$ & $8 \mathrm{dBi}$ \\
\hline $\begin{array}{l}\text { Main lobe } \\
\text { polarization mode }\end{array}$ & $\begin{array}{l}65^{\circ} \mathrm{E} \text { and } \mathrm{H} \\
\text { circular }\end{array}$ & $\begin{array}{l}65^{\circ} \mathrm{E} \text { and } \mathrm{H} \\
\text { vertical }\end{array}$ \\
\hline Frequency band & $10 \mathrm{MHz}$ & $10 \mathrm{MHz}$ \\
\hline Output power & $20 \mathrm{dBm}$ & - \\
\hline Power supply & $\begin{array}{l}12-\mathrm{V} \text { battery or } \\
220 \text { AC source }\end{array}$ & $\begin{array}{l}12-\mathrm{V} \text { battery or } \\
220 \text { AC source }\end{array}$ \\
\hline Preamplifier & - & $25 \mathrm{~dB}$ \\
\hline
\end{tabular}

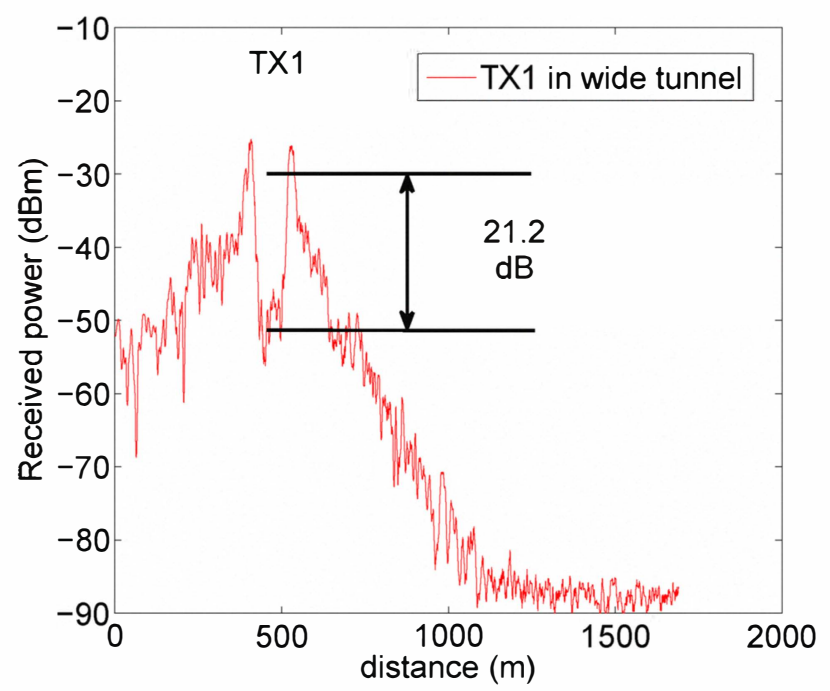

Fig. 3. Coverage of tunnels with transmitter 1 inside tunnels from 0 to $1863 \mathrm{~m}$. Measurements RMS with window length $40 \lambda$.

received signal power of referenced configured antennas on train suffers a strong attenuation. We name such phenomenon as near shadowing. Fig. 3, Fig. 4 and Fig. 5 indicate the near shadowing attenuation depth of received signal from different transmitters inside wide and narrow tunnels, respectively.

As we can see from Fig. 3, Fig. 4 and Fig. 5, two zones can be distinguished during the process of propagation between transmitter and train. When the distance between train and transmitter is shorter than the length of train, the signal is blocked by the carriage so that near shadowing effect occurs. When the train is running out of this region, the coverage recovers. All the detailed parameters of near shadowing effect both in wide tunnel and narrow tunnel have been demonstrated 


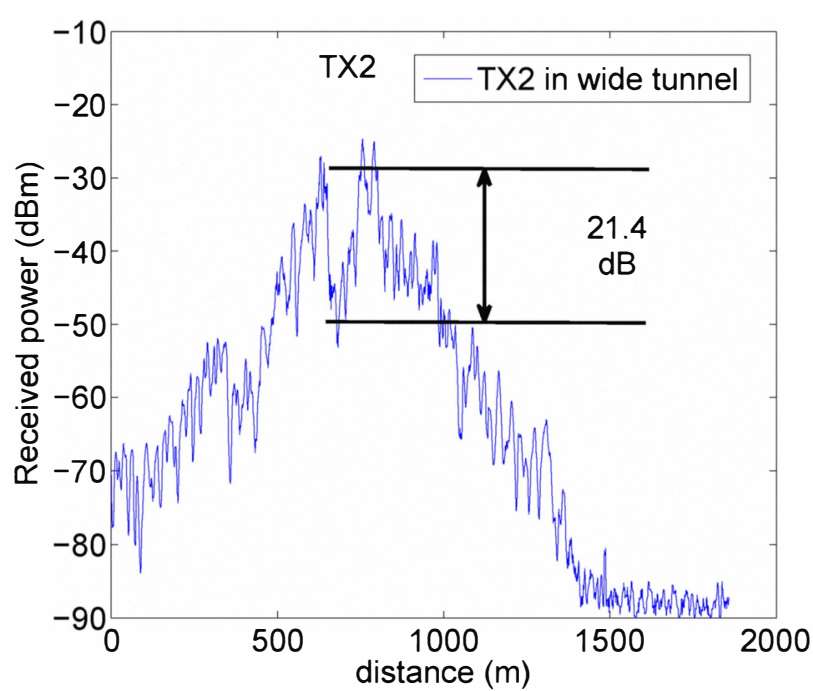

Fig. 4. Coverage of tunnels with transmitter 2 inside tunnels from 0 to $1863 \mathrm{~m}$. Measurements RMS with window length $40 \lambda$.

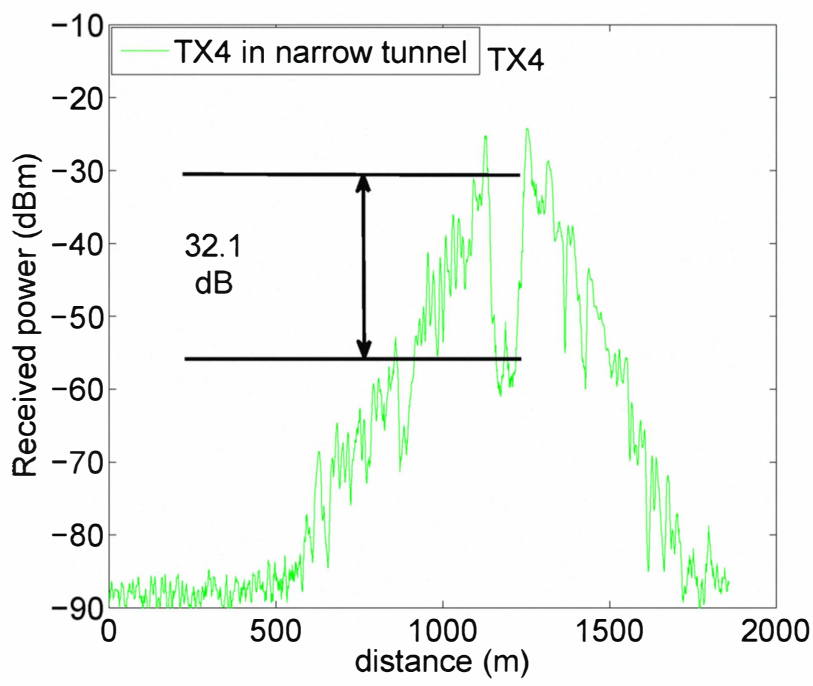

Fig. 5. Coverage of tunnels with transmitter 4 inside tunnels from 0 to $1863 \mathrm{~m}$. Measurements RMS with window length $40 \lambda$.

in Table. II.

TABLE II

COMPARISON OF NEAR SHADOWING CHARACTERISTICS ALONG THE ENTIRE TRIALS. TYPICAL UNDERGROUND TRACK WITH FRONT AND REAR ANTENNAS CONFIGURATION.

\begin{tabular}{ccc}
\hline $\begin{array}{c}\text { Parameters of } \\
\text { Near shadowing }\end{array}$ & $\begin{array}{c}\text { Wide tunnel } \\
\text { (TX 2) }\end{array}$ & $\begin{array}{c}\text { Narrow tunnel } \\
\text { (TX 4) }\end{array}$ \\
\hline $\begin{array}{c}\text { Near shadowing } \\
\text { depth Mean }\end{array}$ & $21.4 \mathrm{~dB}$ & $32.1 \mathrm{~dB}$ \\
$\begin{array}{c}\text { Standard deviation } \\
\text { of fast Fading }\end{array}$ & $5.8 \mathrm{~dB}$ & $6.3 \mathrm{~dB}$ \\
$\begin{array}{c}\text { Near shadowing } \\
\text { region length }\end{array}$ & $\begin{array}{c}\text { Two times of the length of train } \\
(120 \mathrm{~m})\end{array}$ \\
\hline
\end{tabular}

By analyzing near shadowing phenomenon we can get following significant discussions:
- We have measured that the signal received power suffers a severe near shadowing attenuation of up to $21.4 \mathrm{~dB}$ inside the wide tunnel $(9.8 \mathrm{~m} \times 6.2 \mathrm{~m})$ and $32.1 \mathrm{~dB}$ inside the narrow tunnel $(4.8 \mathrm{~m} \times 5.3 \mathrm{~m})$. These values are high and sufficient to produce transmission errors when train is passing the transmitter. Thus it must be considered carefully in the design of a network.

- The length of near shadowing region is approximately two times of the length of the train. This indicates when the train is near the transmitter, the line of sight between the antennas on train and the transmitters inside tunnel has been blocked by the carriage.This phenomenon reveals a practical disadvantage of the current referenced antennas configuration in subway: two antennas installed in the front and in rear part of the train.

- To eliminate near shadowing effect, two engineering suggestions can be considered. One is that the space diversity could be applied in current referenced antennas configuration. More works to verify this solution will be valuable in the future. The other is that different kinds of antennas configurations on-train could be considered: double 2 antennas on both sides of the central car as well as double 4 antennas, two on each side of the front train and two in the rear, for instance.

\section{B. Statistical modeling of near shadowing phenomenon}

As we can see from Fig. 3, Fig. 4 and Fig. 5, two zones can be distinguished during the process of propagation. The first one is the region where the distance between train and transmitter is shorter than the length of train, namely nearshadowing region; the second one is the region where the line of sight between antennas on-train and transmitters recovers, including so-called near field zone and far field zone.

The path loss of the propagation in the second region (near field zone and far field zone) has been proposed in [2],[8] and [9], therefore we need to model the path loss and fast fading of propagation in the near shadowing region in order to make a complete prediction of propagation inside tunnels.

Firstly, we can define that $N L_{\max }$ is the maximum near shadowing loss when there is no line of sight between the transmitter in tunnel and the antennas on cabin. Since such NLOS (non-line of sight) situation results from the block effect of the carriage: the bigger the train, the easier LOS (line of sight) is blocked; the bigger the tunnel, the harder LOS is blocked. Thus, $N L_{\max }$ is proportional to the height and width of the section of the train, but inversely proportional to the height and width of the section of the tunnel. With this analysis, we can give the statistical model on $N L_{\max }$ in this style:

$$
N L_{\max }=a_{1} \times \frac{h}{H}+a_{2} \times \frac{w}{W}
$$

where $w$ and $h$ are the width and height of the cross section of the train, respectively; $W$ and $H$ are the width and height of the cross section of the tunnel, respectively.

Set a group of data $x_{i}, y_{i}, i=1,2, \cdots, n$, if we know that this group of data satisfy some function prototype

$$
\hat{y}(x)=f\left(a_{1}, a_{2}, x\right)
$$


where $a_{1}$ and $a_{2}$ are undetermined coefficients, we can define the optimization by using the principle of Least-squares curve fitting in following way:

$F=\min _{a_{1}, a_{2}} \sum_{i=1}^{n}\left[y_{i}-\hat{y}\left(x_{i}\right)\right]^{2}=\min _{a_{1}, a_{2}} \sum_{i=1}^{n}\left[y_{i}-f\left(a_{1}, a_{2}, x_{i}\right)\right]^{2}$

In our case, using the necessary condition for extremum seeking in multivariate function $F$, we can obtain the equations below:

$$
\left\{\begin{array}{l}
\frac{\partial \sum_{i=1}^{n}\left[N L \max _{i}-f\left(a_{1}, a_{2}, x_{i}\right)\right]^{2}}{\partial a_{1}}= \\
-2 \sum_{i=1}^{n}\left[N L \max _{i}-\left(a_{1} \times \frac{h_{i}}{H_{i}}+a_{2} \times \frac{w_{i}}{W_{i}}\right)\right]\left(\frac{h_{i}}{H_{i}}\right)=0 \\
\frac{\partial \sum_{i=1}^{n}\left[N L \max _{i}-f\left(a_{1}, a_{2}, x_{i}\right)\right]^{2}}{\partial a_{2}}= \\
-2 \sum_{i=1}^{n}\left[N L \max _{i}-\left(a_{1} \times \frac{h_{i}}{H_{i}}+a_{2} \times \frac{w_{i}}{W_{i}}\right)\right]\left(\frac{w_{i}}{W_{i}}\right)=0
\end{array}\right.
$$

Then

$$
\left\{\begin{array}{c}
\left(\sum_{i=1}^{n}\left(\frac{h_{i}}{H_{i}}\right)^{2}\right) a_{1}+\left(\sum_{i=1}^{n}\left(\frac{h_{i}}{H_{i}} \cdot \frac{w_{i}}{W_{i}}\right)\right) a_{2}=\sum_{i=1}^{n}\left(N L \max _{i} \cdot \frac{h_{i}}{H_{i}}\right) \\
\left(\sum_{i=1}^{n}\left(\frac{h_{i}}{H_{i}} \cdot \frac{w_{i}}{W_{i}}\right)\right) a_{1}+\left(\sum_{i=1}^{n}\left(\frac{w_{i}}{W_{i}}\right)^{2}\right) a_{2}=\sum_{i=1}^{n}\left(N L \max _{i} \cdot \frac{w_{i}}{W_{i}}\right)
\end{array}\right.
$$

Solving the equations set upon, the undetermined coefficients can be obtain:

$$
a_{1}=16.1, a_{2}=31.2
$$

Thus, we can get the expression regarding the relative size between the cross-section of the train and the tunnel as follows:

$$
N L_{\max }=16.1 \times \frac{h}{H}+31.2 \times \frac{w}{W}
$$

Therefore, the path loss in near-shadowing region can be described in point slope form as:

$$
P L_{R_{N S}}=\frac{P L_{l}-\left(N L_{\max }+P L_{0}\right)}{l-0} \times d+\left(N L_{\max }+P L_{0}\right)
$$

Where $d$ is the distance between transmitter and receiving antenna, $P L_{l}$ represents the path loss at the point where the distance is the length of train, $l$ is the length of train, $P L_{0}$ is the path loss under LOS condition when the distance between train and transmitter is $0 \mathrm{~m}$, which can be calculated by the model in [2],[8] and [9]. Involving fast fading parameter, we can obtain the complete propagation loss in near shadowing region as follows:

$L_{R_{N S}}=\frac{P L_{l}-\left(N L_{\max }+P L_{0}\right)}{l} d+\left(N L_{\max }+P L_{0}\right)+R(\sigma)$

where $R(\sigma)$ represents a rayleigh distribution with standard deviation $\sigma$. The values of this parameter have been exhibited in Table. II for wide tunnel and narrow tunnel, respectively.

The existing models for the radio-propagation inside subway tunnels are usually presented in a two slope curve [10][11], where the propagation path losses are predicted by two expressions with a different slope parameter [12]. We can describe these models with a general type:

$$
L_{T}= \begin{cases}L_{Z_{N F}}, & d \leq b p \\ L_{Z_{F F}}, & d>b p\end{cases}
$$

where $L_{T}$ represents the propagation loss inside tunnel, $L_{Z_{N F}}$ and $L_{Z_{F F}}$ are the loss in near-field zone and the loss in farfield zone, respectively. $b p$ is the position of breaking point which is the boundary between near-field zone and far-field zone. The slope of the propagation path losses changes before and after $b p$.

Considering the near shadowing phenomenon in practical subway operation, it is essential to add the near shadowing region and corresponding effect into the former model type. Therefore, we can get a complete propagation loss model inside subway tunnel with three expressions in three regions:

$$
L_{T C}=\left\{\begin{array}{cc}
L_{R_{N S}}, & d \leq l_{R_{N S}} \\
L_{Z_{N F}}, & l_{R_{N S}}<d \leq b p \\
L_{Z_{F F}}, & d>b p
\end{array}\right.
$$

where $L_{T C}$ represents the complete propagation loss inside tunnel, $L_{R_{N S}}$ is the loss in near shadowing region and $l_{R_{N S}}$ is the length of near shadowing region which is almost two times of the train length. Substituting equation (9) into equation (11), as well as combining with the propagation model presented in [2], a complete propagation model inside subway tunnel can be obtained as following:

$$
L_{T C}=\left\{\begin{array}{c}
\frac{P L_{l}-\left(N L_{\max }+P L_{0}\right)}{l} d \\
+\left(N L_{\max }+P L_{0}\right)+R(\sigma), \quad d \leq l_{R_{N S}} \\
10 \log _{10}\left[\sum_{i=1}^{m} \sum_{j=1}^{n} \times \sqrt{10^{2 \alpha(i, j)^{h} d}+10^{2 \alpha(i, j)^{v} d}}\right], \\
l_{R_{N S}}<d \leq b p \\
10 \log _{10} \sqrt{10^{2 \alpha(1,1)^{h} d}+10^{2 \alpha(1,1)^{v} d}, \quad d>b p}
\end{array}\right.
$$

where $\alpha(m, n)^{h}$ and $\alpha(m, n)^{v}$ are the loss rate for each $(m, n)$ mode of horizontal and vertical polarizations, respectively. They can be computed as:

$$
\begin{aligned}
& \alpha(m, n)^{h}=4.343 \lambda^{2}\left(\frac{m^{2} \varepsilon_{r 1}}{a^{3} \sqrt{\varepsilon_{r 1}-1}}+\frac{n^{2}}{b^{3} \sqrt{\varepsilon_{r 2}-1}}\right) d B / m \\
& \alpha(m, n)^{v}=4.343 \lambda^{2}\left(\frac{m^{2}}{a^{3} \sqrt{\varepsilon_{r 1}-1}}+\frac{n^{2} \varepsilon_{r 2}}{b^{3} \sqrt{\varepsilon_{r 2}-1}}\right) d B / m
\end{aligned}
$$

where $a$ and $b$ are the horizontal and vertical dimensions of the tunnel, respectively. $\varepsilon_{r 1}$ and $\varepsilon_{r 2}$ are the relative permittivities of the vertical and horizontal walls, respectively.

To validate the proposed model, simulations have been compared with the measurement results. Comparisons of propagation loss between two models and measurement are shown in Fig. 6, Fig. 7 and Fig. 8, where we can see that the new model (spot) gives much better approximation to predict the losses throughout the near shadowing region inside tunnels than the classical model (cross)[11].

\section{CONCLUSION}

A group of measurements carried out in a realistic Madrid subway environment at $2.4 \mathrm{GHz}$ using referenced antennas 
configuration have been reported.

Firstly, based on this measurement, a very important phenomenon named near shadowing effect has been observed. This phenomenon exists in the realistic subway operation and affects the received signal power severely. Then, the cause of this phenomenon has been analyzed; some academic and engineering suggestions have been given in order to change this situation in real operational subway. Finally, the statistical model on near shadowing effect has been built, including which the new propagation loss model with three regions is more complete than previous classical models with two zones. As shown in simulation results, much better fitting effect can be obtained with new model than with classical model both in double track tunnel (9.8 $\mathrm{m}$ width) and single track tunnel (4.8 m width).

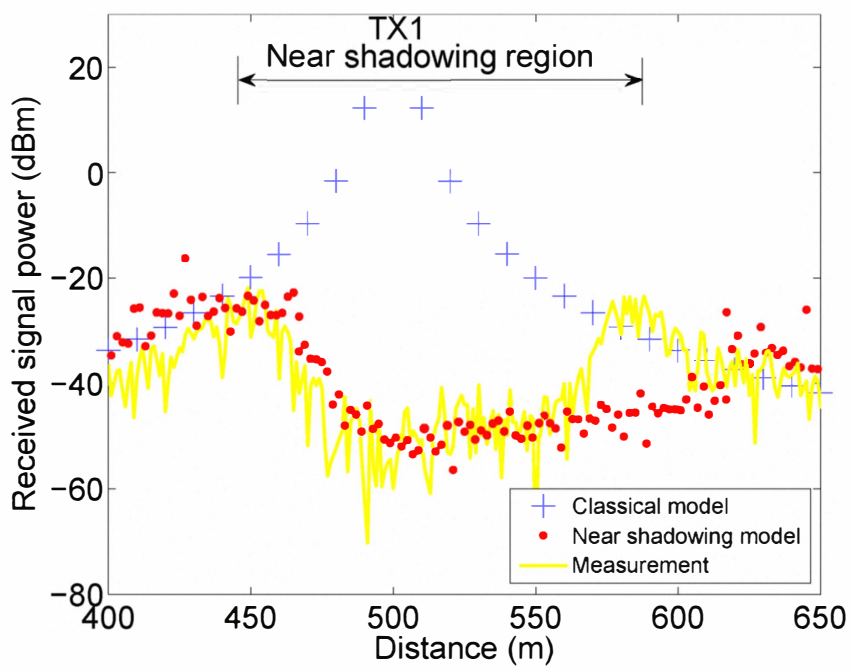

Fig. 6. Comparison of near shadowing phenomenon between new model, classical model and measurement on TX1 inside wide tunnel $(9.8 \mathrm{~m} \times 6.2 \mathrm{~m})$.

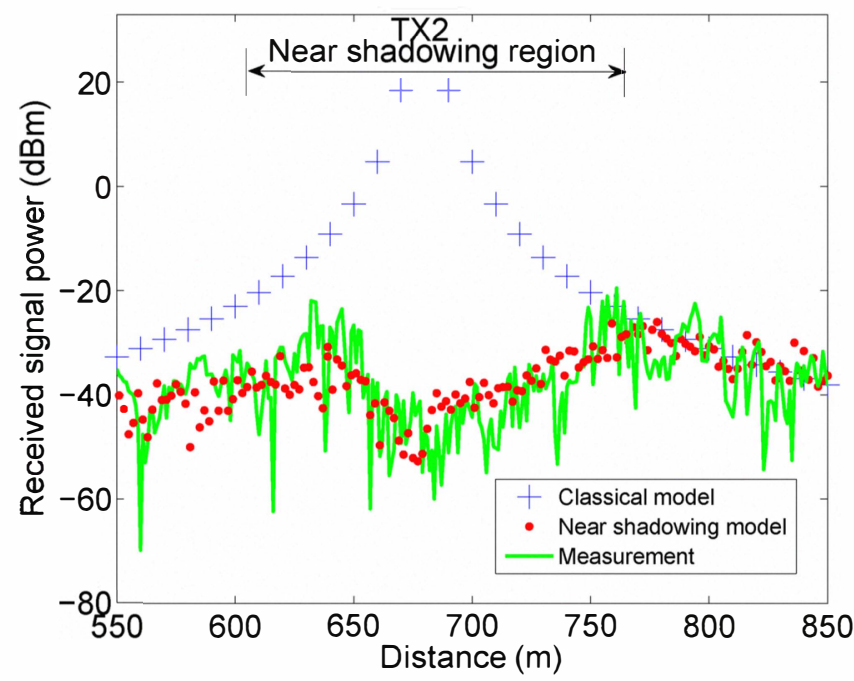

Fig. 7. Comparison of near shadowing phenomenon between new model, classical model and measurement on TX2 inside wide tunnel $(9.8 \mathrm{~m} \times 6.2 \mathrm{~m})$.

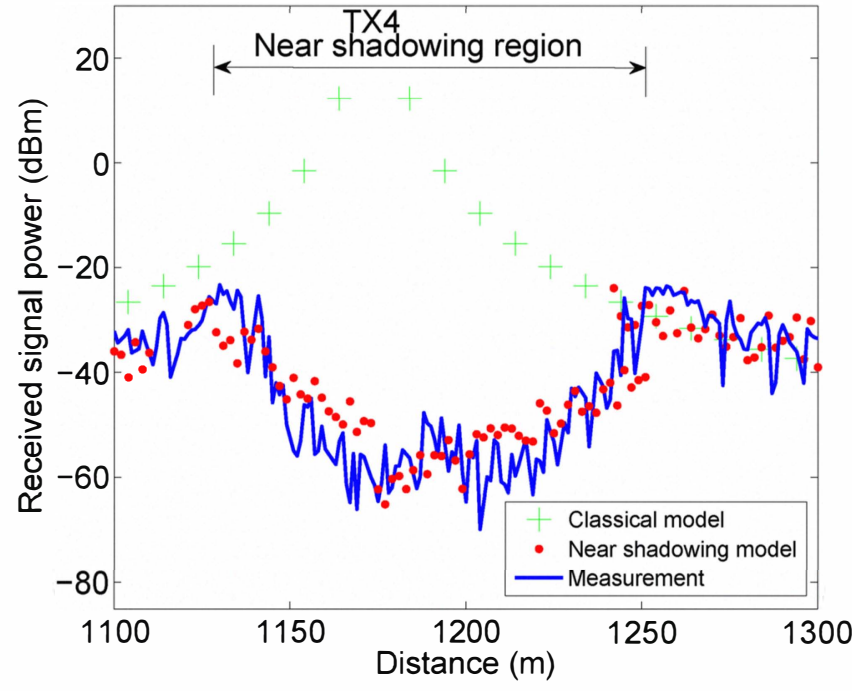

Fig. 8. Comparison of near shadowing phenomenon between new model, classical model and measurement on TX4 inside wide tunnel $(4.8 \mathrm{~m} \times 5.3 \mathrm{~m})$.

\section{REFERENCES}

[1] "IEEE standard for Communications-Based Train Control (CBTC) performance and functional requirements", 30. Dec. 1999.

[2] C. Briso-Rodriguez, J.M. Cruz and J.I. Alonso, J. Xie, Z. Yang, "Measurements and Modeling of Distributed Antenna Systems in Railway Tunnels,'Veh. Technol., IEEE Trans, vol. 56, Issue 5, Part 2, pp. 2870 2879, Sept. 2007.

[3] Y.P. Zhang, "Practical performance of digital cellular system in mass rapid transit environments,"Int. J. Commun. Syst, Vol. 18, pp. 143-157, Dec. 2004.

[4] D. Didascalou, J. Maer, and W. Wiesbeck, "Subway tunnel guided electromagnetic wave propagation at mobile communications frequencies,"IEEE Trans. Antennas Propag., Vol. 49, pp. 1590-1596, Nov. 2001.

[5] M. Lienard and P. Degauque, "Propagation in wide tunnels at $2 \mathrm{GHz}$ : A statistical analysis,"IEEE Trans. Veh. Technol., Vol. 47, pp. 1322- 1328, Nov. 1998.

[6] P. Mariage, M. Lienard, and P. Degauque, "Theoretical and experimental approach of the propagation of high frequency waves in road tunnels,"IEEE Trans. Antennas Propag., Vol. 42, pp. 75-81, Jan. 1994.

[7] Y. P. Zhang, Z. R. Jiang, T. S. Ng, and J. H. Sheng, "Measurements of the propagation of UHF radio waves on an underground railway train,'IEEE Trans. Veh. Technol., Vol. 49, pp. 1342-1347, Jul. 2000.

[8] Y.P. Zhang and Y. Hwang, "Enhancement of rectangular tunnel waveguide model,"Microwave Conf. Proceedings 1997. APMC '97, 1997 AsiaPacific, Vol. 1, pp. 197 - 200, Dec. 1997.

[9] Y.P. Zhang, "Novel model for propagation loss prediction in tunnels,"Veh. Technol. IEEE Trans., Vol. 52, pp. 1308 - 1314, Sept. 2003.

[10] Dudley D. G.; Lienar M.; Mahmud S. F.; Degauque P, "Wireless propagation in tunnels,'IEEE Antennas and Propagation Magazine, Vol. 49, pp. 11C26, Sept. 2007.

[11] Donald G. Dudley and Hsueh-Yuan Pao,"System Identification for Wireless Propagation Channels in Tunnels,"IEEE Trans. Antennas Propag., Vol. 53, pp. 2400 - 2405, Aug. 2005.

[12] J. Alonso ; S. Capdevilla ; B. Izquierdo ; J. Romeu, "Propagation measurements and simulations in tunnel environment at $5.8 \mathrm{ghz}$,"Antennas Propag. Society, IEEE Int. Symp., pp.1 - 4, pp. 11C26, 2008. 